VIII ${ }^{\text {ème }}$ Journées Génie Civil - Génie Côtier, Compiègne, 7-9 septembre 2004

\title{
Proposition d'une méthode d'essai destructive in-situ pour la caractérisation de l'intégrité des enrochements naturels utilisés dans les ouvrages hydrauliques
}

\author{
S. Dupray ${ }^{(a)}$, J.L. Durville ${ }^{(b)}$, J.P. Latham ${ }^{(c)}$ \\ (a)chef de section, Ministère de l'Équipement, des Transports, de l'Aménagement du territoire, \\ du Tourisme et de la Mer - CETE de Lyon, 25 avenue F Mitterrand \\ BP 1 - 69674 BRON cedex \\ sebastien.dupray@equipement.gouv.fr \\ (b) chargé de missions, Ministère de l'Équipement, des Transports, de l'Aménagement du territoire, \\ du Tourisme et de la Mer-CETE de Lyon, 25 avenue F Mitterrand \\ BP 1 - 69674 BRON cedex \\ jean-louis.durville@equipement.gouv.fr \\ (c)enseignant chercheur, Imperial College, Royal School of Mines Prince Consort road, \\ SW7-2BP Londres \\ j.p.latham@imperial.ac.uk
}

\section{Résumé:}

La qualité des enrochements naturels utilisés pour la construction des ouvrages hydrauliques du type digue est importante car elle a une forte influence sur leur stabilité, leur dimensionnement et leur politique de maintenance. L'intégrité est considérée comme 'essentielle' par les professionnels mais ne fait l'objet d'aucune méthode de caractérisation satisfaisante. Cet article présente une méthode d'essai destructive in-situ qui permet de caractériser efficacement des lots présentant différents types de défauts et d'estimer les modifications de la blocométrie lors de manipulations. Il est proposé d'utiliser cet essai et la méthode d'analyse associée comme un outil de prévision de la blocométrie à prendre en compte lors du dimensionnement, notamment pour des sites dépourvus d'enrochements de première qualité. Les résultats ne peuvent être utilisés sans l'autorisation écrite des auteurs.

\begin{abstract}
:
The quality of natural armourstone used as building material of hydraulic structures such as breakwaters is an important issue since it has a significant influence on their stability, their design and their maintenance policy. Professionals consider integrity as 'essential' but no satisfactory test method is available yet. This paper presents an in-situ test method as a means to quantitatively and efficiently differentiate consignments affected by different types of defects and to estimate the modifications of the mass distribution curve during routine handling. It is proposed to use this test method and the associated analysis to predict the mass distribution to account for at design stage, especially in region deprived of good quality armourstone source. Results cannot be used prior to written authorisation of the authors.
\end{abstract}

Mots clés:Enrochement, intégrité, discontinuité, méthode d'essai, ouvrage hydraulique, digue

Keywords: Armourstone, integrity, discontinuity, test method, hydraulic structure, breakwater 


\section{La qualité des enrochements utilisés dans les ouvrages hydrauliques}

\subsection{Les enrochements naturels dans les ouvrages hydrauliques}

Des ouvrages hydrauliques sont requis pour procurer un abri à des navires ou à des installations, une retenue d'eau utilisée comme réservoir ou source d'énergie, une voie de transport ou d'irrigation. L'enrochement est largement utilisé comme matériau de construction de ces ouvrages dans les différentes parties qui les composent : noyau, filtre, carapace, butée de pied. Sa souplesse d'utilisation et sa disponibilité à proximité du site de construction en font un matériau privilégié.

Les caractéristiques des blocs : densité de la roche, forme,... et celles du matériau granulaire composé de ces blocs : masse médiane M50 ou diamètre nominal médian Dn50, gradation Dn85/Dn15, imbrication potentielle (fortement influencée par la mise en œuvre), porosité, épaisseur,... interviennent dans la conception de deux façons. Lors d'une conception basée sur le dimensionnement hydraulique, il s'agit de trouver la source d'enrochement aux propriétés requises, qui peut se trouver assez loin du lieu de mise en œuvre, entraîner des impacts environnementaux et des coûts non-négligeables liés au transport et conduire à l'utilisation d'autres matériaux comme des blocs artificiels préfabriqués en béton. Alternativement, l'utilisation de gisements locaux peut être recherchée en intégrant en amont les caractéristiques de l'enrochement disponible lors d'une conception basée sur la fourniture .

\subsection{La qualité des enrochements}

La période de vie de l'enrochement peut être décomposée en une période dite de 'court terme' qui correspond aux phases d'extraction, de production, de stockage, de manipulation, de transport, de mise en œuvre et une période dite de 'long terme'. Celle-ci correspond à la situation en service : une période transitoire d'ajustement de l'ouvrage sous l'action des premières tempêtes suivie d'une situation permanente entrecoupée d'évènements tels que les tempêtes. Durant ces périodes, l'enrochement est exposé à une succession de sollicitations liées à la méthode de construction, au type d'ouvrage et à son exposition aux actions hydrauliques et climatiques.

Ainsi, l'enrochement doit avoir des propriétés complémentaires à celles mentionnées au paragraphe 1.1, telles que des propriétés de résistance mécanique, de durabilité face à des phénomènes d'abrasion, de gel-dégel... Cet article s'intéresse précisément à la résistance mécanique des enrochements qui a été identifiée comme une caractéristique 'essentielle' lors d'une étude conduite auprès de professionnels français et britanniques .

L'enrochement peut présenter des ruptures lors du court terme. Elles sont liées aux manipulations qui peuvent atteindre la dizaine et qui produisent chacune des impacts ou chocs sur le matériau. En service, elles sont provoquées par l'impact de blocs en mouvement dans la carapace ou dues aux mouvements du bloc lui-même, particulièrement dans les digues reprofilables. Les mécanismes de rupture, leurs effets et les méthodes pour les quantifier ou s'en prémunir restent mal connus.

\subsection{Ruptures des enrochements}

On admet en général que les blocs d'enrochement présentent deux types de ruptures qualifiées de rupture mineure ou 'broyage' et de rupture majeure ou 'fendage'. La rupture mineure correspond à l'écrasement des arêtes et des coins des blocs. Elle se produit dans la matrice 
rocheuse et génère des fragments pesant au plus quelques dizaines de kilogrammes comme illustré à la Figure 1a. Il est généralement admis que les essais de caractérisation de la matrice rocheuse permettent d'évaluer le risque d'apparition de ce phénomène. La rupture majeure est contrôlée par les défauts préexistants dans le bloc qui peuvent se propager et conduire au fendage en un nombre limité de blocs comme montré à la Figure 1b. En particulier, l'origine des discontinuités : géologiques ou liées à l'exploitation, leur position, leur nombre, leur extension dans le bloc contrôlent la résistance des blocs d'enrochement à la rupture le long de ces discontinuités.

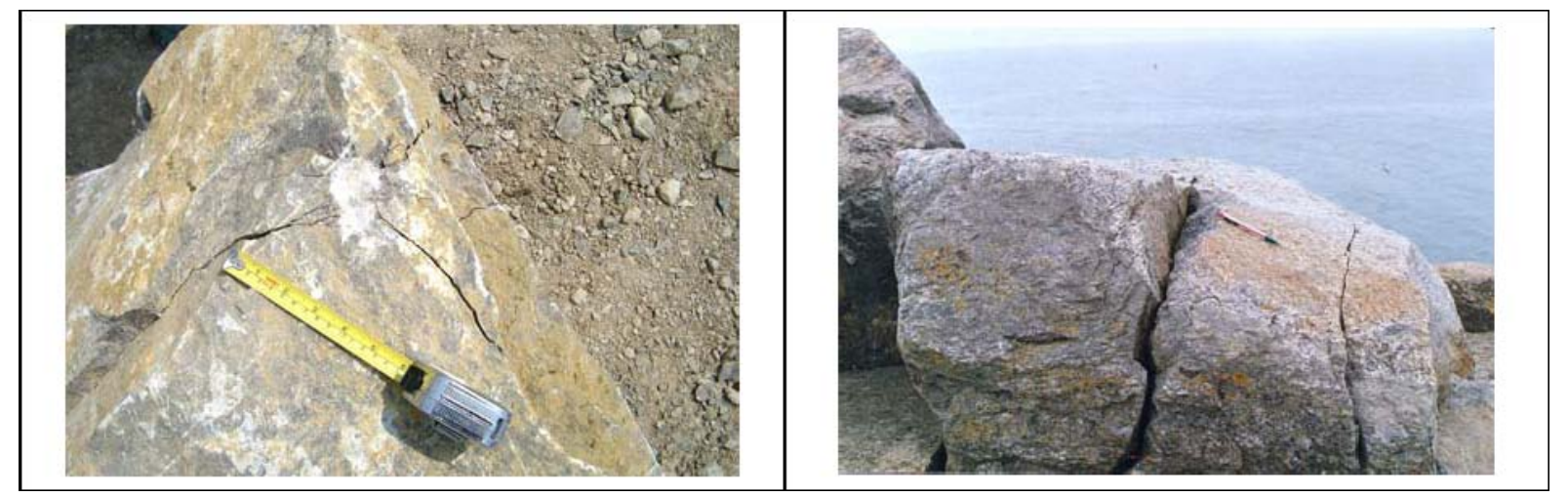

Figure 1. Exemple de ruptures observables sur un bloc d'enrochement (a) mineure sur un bloc sur stock (b) majeure sur un bloc dans un ouvrage avec des discontinuités d'origine géologique

Les observations tendent à montrer que la limite entre rupture majeure et rupture mineure se situe pour une perte de masse $L m$ d'environ $10 \%$. $L m$ est calculé par l'équation (1) où $M i$ est la masse initiale du bloc, $M f$ la masse du plus gros morceau de bloc résultant de la dégradation.

$$
L=\left(M_{i}-M^{*}\right) / M_{i}(1)
$$

\section{Intégrité des enrochements}

\subsection{Double définition de l'intégrité}

La capacité de l'enrochement à résister aux ruptures majeures est généralement désignée par 'intégrité'. Il faut cependant distinguer deux types d'intégrité. D'une part, il y a l'intégrité d'un bloc d'enrochement qui est sa capacité à ne pas présenter de rupture majeure pendant le court et le long terme. D'autre part, il y a l'intégrité de l'enrochement, en tant que matériau granulaire, qui est son aptitude à ne pas présenter de changement significatif de ses propriétés géométriques, à savoir la taille médiane ou moyenne et la gradation, lors du court et du long terme.

Pour des raisons pratiques relatives à la taille des blocs d'enrochement et à la taille d'un échantillon représentatif, la détermination de l'intégrité ne peut se faire qu'à partir d'essais sur des blocs individuels. Ainsi, deux approches existent.

La première est l'approche statistique qui consiste à définir une proportion acceptable de blocs qui peuvent rompre pendant l'essai, effectué sur un lot représentatif ; c'est l'approche traditionnelle. La deuxième approche que nous proposons est l'approche dite blocométrique qui consiste à étudier les variations de la courbe blocométrique pendant l'essai et à les calibrer par référence à des variations mesurées sur site. Cette deuxième approche permet de déterminer de 
façon raisonnée le seuil à prendre dans la méthode statistique à partir d'exigences spécifiées sur Dn50 en intégrant les sollicitations appliquées à l'enrochement comme détaillé au paragraphe 4.

\subsection{Méthodes de caractérisation de l’intégrité}

La caractérisation de la résistance mécanique des enrochements peut-être réalisée à différents stades de leur cycle de vie : pendant sa production, au moment de la construction ou en service. Différentes méthodes sont rapportées dans la littérature et sont présentées ci-dessous.

L'observation visuelle de l'enrochement sur le lieu de production est une méthode de contrôle qualité. Elle reste qualitative et peut conduire à des différences notables de jugement entre contrôleurs. L'évaluation des performances pendant la construction ou en service permet d'évaluer l'intégrité in-situ. Mais la prise en compte des variations dues aux conditions de production, de construction et de service est rarement possible. Les mesures physiques comme l'auscultation sonique sont couramment utilisées. Néanmoins, si la détection des défauts par cette méthode est reconnue, sa capacité à mesurer l'intégrité reste discutée . Les essais en laboratoire sur échantillons permettent de caractériser la matrice rocheuse, mais pas l'intégrité de l'enrochement. Les essais destructifs in-situ sont une autre solution qui est fait l'objet de ce papier.

L'essai de chute ou essai d'impact in-situ est recommandé cependant une étude préliminaire en 2002 a montré qu'il présente un forte une variabilité essentiellement liée aux conditions d'essais notamment la rotation du bloc pendant la chute et la surface d'impact non standard. De plus, la définition de la hauteur de chute qui vaut 3 mètres en Europe et 0,5·Dn50 aux USA n'est pas justifiée dans la littérature. Aucune relation entre les résultats de l'essai et les dégradations effectives en construction ou en service n'est disponible.

Törum propose un essai d'écrasement in-situ qui consiste à soumettre les prises d'essai à des impacteurs de différentes tailles, qui choient de hauteurs variables en sorte qu'une large gamme d'énergie d'impact peut être couverte. Cette configuration permet un meilleur contrôle des conditions d'essai mais nécessite un grand nombre d'essais. De plus, l'analyse est basée sur une approche développée pour les granulats qui suppose l'homogénéité du bloc d'enrochement et ne permet donc pas de distinguer l'effet des discontinuités de celui de la matrice rocheuse lors des ruptures.

\section{Proposition d'une nouvelle méthode d'essai}

\subsection{Motivations et objectifs}

Pour les deux approches de conception présentées au paragraphe 1.1, il est nécessaire soit de s'assurer que l'enrochement ne présentera pas de rupture au-delà d'une valeur acceptable soit de déterminer les changements de la courbe blocométrique afin de les intégrer dans le dimensionnement de l'ouvrage. Aucune méthode ne permet actuellement de réaliser quantitativement ces deux objectifs.

Ainsi, une action de recherche est en cours avec pour objectifs de développer une méthode d'essai in-situ qui permette (i) de comparer objectivement l'intégrité de lots d'enrochements (ii) d'évaluer la contribution de la matrice rocheuse et des discontinuités (iii) de proposer un modèle de prédiction des changements de la blocométrie pendant la construction ou en service (iv) et ainsi de fournir une référence pour l'évaluation de méthodes non-destructives de contrôle. 


\subsection{L'essai de fendage in-situ (FSST)}

L'essai proposé dans le cadre de cette recherche consiste à faire choir d'une hauteur $H d$ un impacteur en acier de masse $M I$, sur le bloc à tester, qui est placé sur une enclume de masse $M A$ de même roche que le bloc testé. Les blocs d'enrochements sont pesés avant l'essai ainsi que tous les fragments supérieurs à $10 \mathrm{~kg}$ obtenus après l'essai. La configuration de l'essai est présentée à la Figure 2.

La masse de l'enclume est égale à la masse extrême supérieure (EUL) de la blocométrie définie selon la norme NF EN 13383-1 ${ }^{10}: M A=E U L$. Le bloc à tester est positionné au centre de l'enclume. S'il possède une discontinuité, détectée visuellement ou à l'aide de mesures soniques, celle-ci est placée verticalement. La hauteur de chute vaut $2 \mathrm{~m}$.

Les principes de détermination de la hauteur de chute sont les suivants : seuls les blocs possédant une discontinuité présentent une rupture majeure pendant l'essai ; la force maximale d'impact appliquée au bloc pendant l'essai peut être déterminée d'après Knight à partir des propriétés mécaniques de l'impacteur, du bloc et de l'enclume en fonction de la hauteur de chute ; la contrainte maximale dans le plan vertical du bloc peut être déterminée d'après Shipway à partir de la force d'impact appliquée et de l'épaisseur du bloc entre les points de contact avec l'enclume et l'impacteur. La résistance à la traction de la matrice minérale a été déterminée en laboratoire sur échantillon. La contrainte maximale admissible dans un bloc intact pendant l'essai de fendage a été choisie à $80 \%$ de la valeur caractéristique de résistance à la traction du site de plus faible résistance.

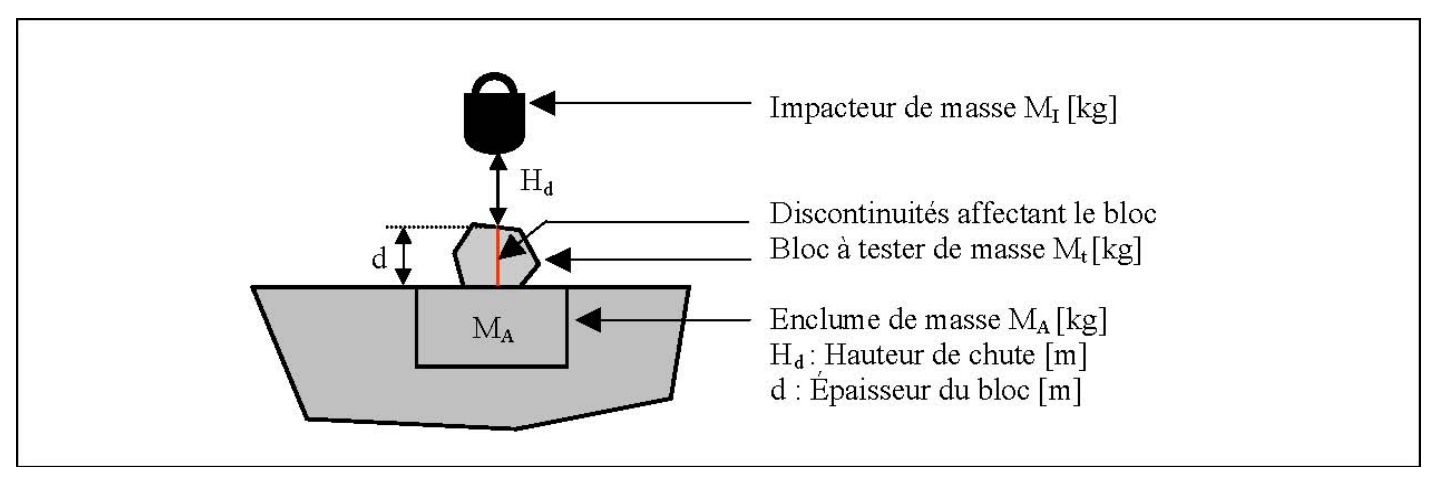

Figure 2. Description de l'essai de fendage in-situ proposé

\section{Résultats préliminaires}

Une campagne d'essais a été menée sur deux gisements différents où deux méthodes d'exploitation différentes sont utilisées. Les caractéristiques ont été déterminées au laboratoire sur des échantillons prélevés sur l'enrochement testé sur site et sont rassemblées au tableau 1. 
Tableau 1 : Caractéristiques des roches

\begin{tabular}{|l|l|l|}
\hline & Site A & Site B \\
\hline Type de gisement & Sédimentaire & Magmatique \\
\hline Type d'exploitation & Granulat & Pierre Ornementale \\
\hline Origine de l'enrochement & $\begin{array}{l}\text { Directement du tir } \\
\text { sans contrôle qualité }\end{array}$ & $\begin{array}{l}\text { Issu des stocks } \\
\text { après contrôle qualité }\end{array}$ \\
\hline Classe granulaire [kg] NF EN 13383-2 & $1000-3000$ & $1000-3000$ \\
\hline $\begin{array}{l}\text { Masse volumique [1000kgm-3] NFP } \\
94410-2\end{array}$ & $2.7 \pm 0.0^{*}$ & $2.7 \pm 0.0^{*}$ \\
\hline Porosité [\%] NFP 94410-3 & $0.7 \pm 0.4^{*}$ & $0.13 \pm 0.1^{*}$ \\
\hline Rc [MPa] NFP 94420 & $162.4 \pm 40.3^{*}$ & $234.0 \pm 18.1^{*}$ \\
\hline Rtb [MPa]* NFP 94422 & $7.4 \pm 2.3^{*}$ & $10.3 \pm 1.0^{*}$ \\
\hline E [GPa] NFP 94425 & $80.9 \pm 4.6^{*}$ & $75 \pm 1.3^{*}$ \\
\hline$v[-]$ NFP 94425 & $0.3 \pm 0.0^{*}$ & $0.25 \pm 0.0^{*}$ \\
\hline Vl sur éprouvette [ms-1] NFP 94411 & $6199.5 \pm 160.2^{*}$ & $5747.2 \pm 120.0^{*}$ \\
\hline
\end{tabular}

* moyenne \pm écart type

Pour les deux sites, un lot d'au moins 200 blocs issus du même gisement a été prélevé. L'observation visuelle a permis de classer l'enrochement par type de discontinuité (voir la Figure 3) ainsi que la composition du lot global. A partir des essais de laboratoires (voir le Tableau 1), la hauteur de chute a été calculée en utilisant la valeur moyenne de la résistance à la traction, Rtb, du site A comme indiqué au paragraphe 3.2. La hauteur de chute ainsi déterminée vaut 2 mètres.

\section{1.Étude ${ }^{\circ} 1$ : effet des discontinuités sur les résultats de l'essai de fendage in situ.}

Des lots de même taille ne présentant qu'un seul type de discontinuité ont été composés et soumis à l'essai de fendage in-situ. La Figure 4 présente la blocométrie initiale testée ( ) et les blocométries des lots présentant des discontinuités $\left(\times_{\tilde{,}, \tilde{}}^{\sim}\right)$.
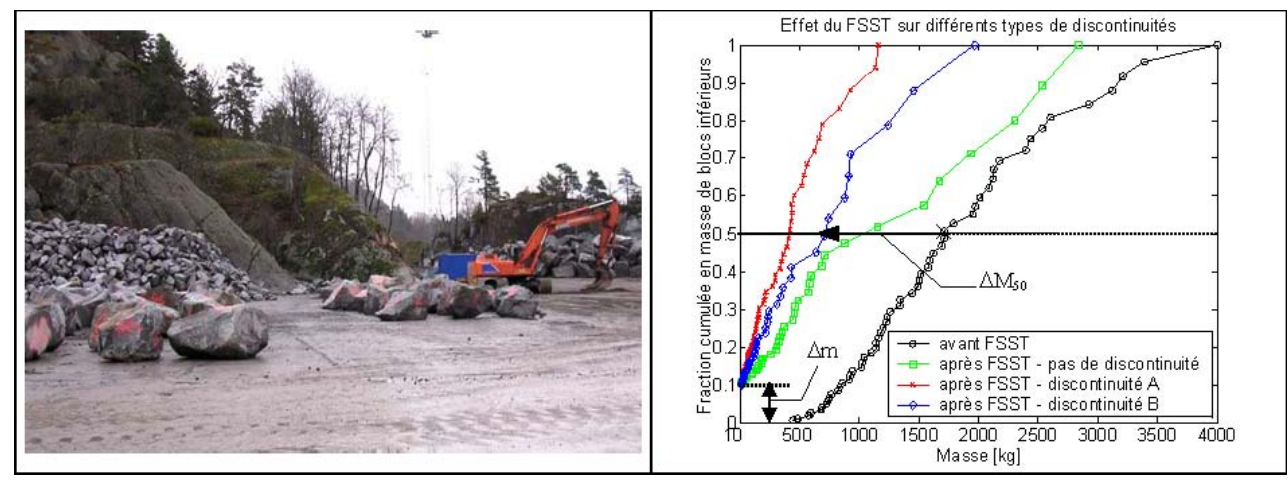

Figure 3. Exemple de préparation de Figure 4. Exemple de modification de la l'échantillon-classement par type de blocométrie pendant le FSST pour différents discontinuité types de discontinuités 
Sur la courbe blocométrique, le décalage de l'ordonné, mesuré à 10kg (voir figure 4), est noté $\Delta m$. C'est une caractéristique des propriétés de la matrice minérale. La convergence de toutes les courbes vers la même valeur confirme que la matrice rocheuse est prise en compte de façon équivalente pour les différents lots et que seul l'effet des discontinuités est à l'origine de la différence entre les courbes après essai.

Le défaut d'intégrité est caractérisé par le décalage de la blocométrie vers des masses plus faibles et par la décroissance relative du poids médian, $\Delta M 50$, calculé à l'aide de l'équation (2). Ce décalage dépend du type de discontinuité (D1 à D4) présenté par l'enrochement (voir tableau 2).

$$
{ }^{\mathrm{M}} 50=\left({ }^{\mathrm{M}} 50 \mathrm{i}^{-\mathrm{M}} 50 \mathrm{f}\right) /{ }^{\mathrm{M}} 50 \mathrm{i}
$$

M50i est la masse médiane initiale de l'enrochement, M50f est la masse médiane initiale de l'enrochement résultant de l'essai.

Tableau 2 : Résultats des essais de fendage in-situ

\begin{tabular}{|l|l|l|l|l|l|l|}
\hline & $\begin{array}{l}\Delta m \\
{[\%]}\end{array}$ & $\Delta M 50[\%]$ & $\begin{array}{l}\Delta M 50 \\
{[\%]}\end{array}$ & $\begin{array}{l}\Delta M 50 \\
{[\%]}\end{array}$ & $\begin{array}{l}\Delta M 50 \\
{[\%]}\end{array}$ & $\begin{array}{l}\Delta M 50 \\
{[\%]}\end{array}$ \\
\hline & & Sans discontinuité & D1 & D2 & D3 & D4 \\
\hline $\begin{array}{l}\text { Site } \\
\text { A }\end{array}$ & 10 & 29 & 76 & 58 & 55 & - \\
\hline $\begin{array}{l}\text { Site } \\
\text { B }\end{array}$ & 5 & 20 & 46 & - & - & 24 \\
\hline
\end{tabular}

\section{2.Étude $\mathrm{n}^{\circ}$ 2: Calibration de l'essai de fendage in-situ}

Un lot de 100 blocs de composition identique à celle observée sur le prélèvement initial a été réalisé puis divisé en deux populations de même composition. Le premier a été soumis à l'essai de fendage. Le second a été manipulé sur le site avec un chargeur en suivant les procédures utilisées pour la mise en dépôt, visible à la Figure 5 après manipulations. La Figure 6 présente les blocométries : initiale, après FSST sur le premier échantillon et après manipulations du deuxième échantillon.

Le tableau 3 présente les résultats de la calibration pour les deux sites $\mathrm{A}$ et $\mathrm{B}$, à savoir les résultats du FSST et des manipulations réalisés sur des échantillons de même composition.
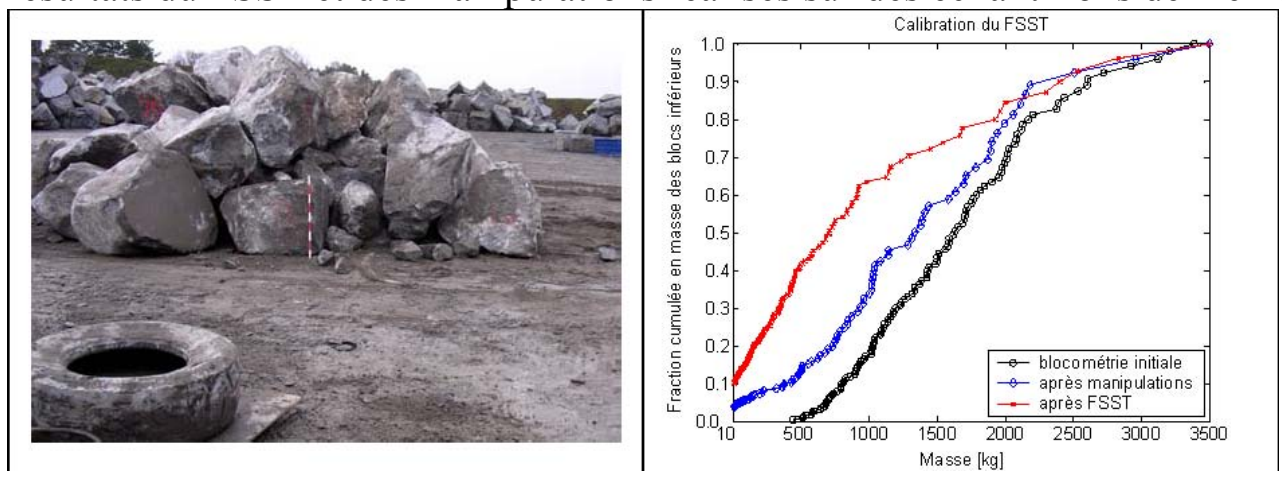

Figure 5. Exemple de dégradation d'un lot Figure 6. Exemple de calibration-après manipulations - Noter la bascule au 1 blocométrie : initiale $\circ$, après essai $\times$; après plan manipulations $\diamond$ 
Tableau 3 : Résultats bruts de la calibration

\begin{tabular}{|l|l|l|l|l|}
\hline & \multicolumn{3}{|l|}{ Site $\mathrm{A}$} & \multicolumn{3}{l|}{ Site $\mathrm{B}$} \\
\hline Composition $*:$ & \multicolumn{3}{|l|}{$\mathrm{H} \approx 40 \% ; \mathrm{G} \approx 50 \% ; \mathrm{OC} \approx 10 \%$} & \multicolumn{1}{l|}{$\mathrm{H} \approx 70 \% ; \mathrm{G} \approx 20 \% ; \mathrm{OC} \approx 10 \%$} \\
\hline Essai de fendage [\%] & $\Delta m=10.0$ & $\Delta M 50=59.0$ & $\Delta m=5.0$ & $\Delta M 50=27.0$ \\
\hline Manipulations : $2[\%]$ & $\Delta m=5.0$ & $\Delta M 50=14.0$ & $\Delta m=3.5$ & $\Delta M 50=9.0$ \\
\hline Manipulations : $4[\%]^{* *}$ & $\Delta m=3.0$ & $\Delta M 50=7.0$ & $\Delta m=1.5$ & $\Delta M 50=5.0$ \\
\hline
\end{tabular}

*proportion en nombre de blocs, $H$ : homogène, $G$ : discontinuité géologique, $O C$ : fissure ouverte valeur non cumulée

\subsection{Modèle de dégradation}

La forme des courbes blocométriques peut être schématisée en considérant les diamètres nominaux, comme indiqué à la Figure 7 . Les surfaces, $\Delta A$, ainsi créées entre la courbe granulométrique initiale et la courbe après essai de fendage et un certain de nombre de manipulations sont notées respectivement $\triangle A T e s t$ et $\triangle A H$.

Dans les théories de comminution et de fragmentation, $\Delta A$ est utilisé comme indicateur et est relié à l'énergie totale appliquée rapportée à la masse d'enrochement testé, $E s$, et à une dimension caractéristique de l'enrochement $X_{14}$, pour déterminer une résistance caractéristique à la fragmentation notée $C s$. Lu et Latham proposent la relation (3).

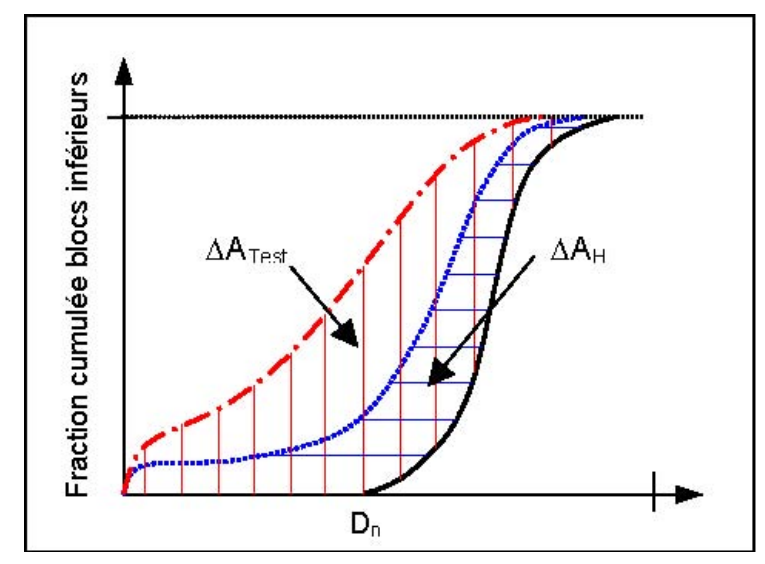

Figure 7. '-' courbe initiale ; '-.-' courbe après essai de fendage; '...' courbe après manipulation(s)

$$
E s=C s\left(\triangle A \sqrt{X_{0}}\right)
$$

Pour l'analyse de l'essai de fendage (voir section 4.1), la relation (3) permet de déterminer la résistance caractéristique $\mathrm{Cs}$ à partir de $E s$ connue, de $X o$ et de $\Delta A$ mesurés. La calibration (voir section 4.2) permet de déduire l'énergie appliquée lors des manipulations, Es, à partir de Cs qui est déterminée sur la moitié de l'échantillon soumis au FSST, de Xo et de $\Delta A$ mesurés lors de la calibration de l'autre moitié de l'échantillon. 
Un modèle de prédiction des changements de la granulométrie, basé sur les travaux de Lu et 14

Latham est en cours de développement pour différencier la part due aux ruptures majeures et mineures. Pour le comportement à court terme, ce modèle est en cours de validation à l'aide des résultats expérimentaux obtenus sur les sites A et B. Pour le long terme, il est proposé de relier l'énergie spécifique aux mouvements des blocs en service grâce aux travaux de Törum et à une recherche en cours à l’Université de Plymouth ${ }^{15}$.

\section{Conclusion}

Cette recherche a permis d'identifier les méthodes d'essais destructives in-situ comme les seules méthodes capables de caractériser objectivement l'intégrité des enrochements utilisés dans les ouvrages hydrauliques. Un essai de fendage in-situ est proposé ainsi qu'une méthode d'analyse qui permet de différencier la contribution respective de la matrice minérale et des discontinuités. Il permet de prédire les changements de blocométrie à court terme. Deux campagnes d'essai ont permis de valider sa capacité à distinguer l'effet des discontinuités de celui de la matrice rocheuse et ainsi de fournir des résultats nécessaires à la validation d'un modèle de dégradation.

\section{Remerciements}

Cette recherche est financée par le CETMEF et le LCPC (France) et Environment Agency (Royaume-Uni), réalisée sous la direction de JL Durville (CETE de Lyon) et de JP Latham (Imperial College). JJ Trichet (CETMEF ), J Simm (HR Wallingford) et les carrières sont remerciées pour leur implication dans ce projet.

\section{Références}

1 Sigurdarson, S. (2002). The Bakkafjordur Breakwater. PIANC WG40. 11 p.

2 Dupray, S., Durville, J.L., Latham, J-P. (2003). Armourstone integrity - towards a full scale destructive testing. Proc Industrial Minerals and Building Stones 2003. Istanbul. 11 p.

3 Latham, J-P. (1995). The drop test for armourstone integrity. in River, coastal and shoreline protection. CRA Thorne et al., Wiley, p. 481-499.

4 Marcus, D. (1995). Problems and improvement of armor stone quality for coastal structures. in River, coastal and shoreline protection. C.R.A. Thorne Wiley et al., p. 532-542.

5 Erikson, R. (1993). Evaluation of limestone and dolomite armor stone durability from observations in the great lakes region. in Rock for erosion control. ASTM, p. 88-94.

6 Niese, M., Laan, G., Verhoef, P. (1990). Quality assessment of large armourstone using an acoustic velocity analysis. in Bulletin of the International Association of Engineering Geology, $\mathrm{p}$. 57-65.

7 Perrier, J. (2002). Méthode de diagnostic des protections de berge en enrochements de la vallée du Rhône. à paraître, $22 \mathrm{p}$.

8 LCPC (1989). Les enrochements. presses $d u$ LCPC, Paris. 106 p.

9 Dupray, S. (2002). Armourstone integrity research, Drop-test Preliminary field study. Progress report Imperial College - Earth Science and Engineering Department London 83 p.

10 AFNOR (2003). NF EN 13383-1 Enrochements - spécifications. Indice P18-661-1. 45 p. 
11 Törum, A. Krogh, S. (2000). Berm breakwater-stone quality. SINTEF Trondheim 80 p.

12 Knight, C. Swain, M, Chaudhry, M. (1977). Impact of small steel spheres on glass surfaces. Journal of Material Sciences. (12). p. 1573-1586.

13 Shipway, P., Hutchings, I. (1993). Fracture of brittle spheres under compression and impact loading I. elastic stress distribution. Philosophical Magazine A 67(6). p. 1389-1404.

$14 \mathrm{Lu}$, P., Latham, J-P. (1998). A model for the transition of block sizes during fragmentation blasting of rock masses. International Journal of Blasting and Fragmentation. (2) p. 341-368.

15 Trmal, C. (2004). Study of mechanical loadings applied to natural armourstone in cover layer of breakwater under wave action. PRCE501-MsC Research, Faculty of Technology -Civil Engineering Department, Plymouth University. 3 p. 